\title{
Consideraciones sociales y filosóficas para la investigación cualitativa de las creencias irracionales
}

\author{
Oscar Gómez González ${ }^{1}$ \\ Universidad de Guadalajara
}

\section{Ensayo}

Material original autorizado para su primera publicación de la Journal de Ciencias Sociales, Revista Académica de la Facultad de Ciencias Sociales de la Universidad de Palermo.

Recepción: 23-11-2020

Aceptación: 11-03-2021

Resumen: Las creencias irracionales son un constructo psicológico propuesto por Albert Ellis, el cual refiere a un tipo de cogniciones dogmáticas e inflexibles que una persona mantiene sobre valores y metas personales. El principal interés sobre estas creencias es que generan afecciones a la salud mental como estrés, ansiedad y depresión, además de tender a desarrollar en la persona, un comportamiento disfuncional, lo que dificulta el logro de metas. Este constructo se ha estudiado mayormente desde una metodología cuantitativa con resultados que han permitido confirmar diversas hipótesis de interés propiamente psicológicas, específicamente para la psicología cognitivo conductual. Sin embargo, estas creencias tienen un origen en el dinamismo entre el individuo y la sociedad, además de presentar algunas dificultades de orden filosófico, por lo que estudiar estas creencias desde una metodología cualitativa puede aportar a estudios sociológicos desde una perspectiva weberiana, y a profundizar en la comprensión fundamental de las creencias irracionales, a través una reflexión plenamente filosófica, particularmente con elementos de la filosofía estoica, la fenomenología de Husserl y los imperativos kantianos. Se abordan teorías desde distintos ejes a propósito de las consideraciones psicológicas, metodológicas, sociológicas y filosóficas, para la comprensión de la relevancia científica de realizar investigación con enfoque cualitativo y la necesidad de integrar en el proceso una reflexión propiamente sociológica y filosófica.

Palabras clave: creencias irracionales; investigación cualitativa; sociología; fenomenología.

\footnotetext{
${ }^{1}$ Licenciado en filosofía por la Universidad América Latina. Estudiante en la Maestría en Psicología, con orientación en Calidad de Vida y Salud, en la Universidad de Guadalajara.

Correo electrónico: oscargomezcontacto@gmail.com
} 


\section{Social and philosophical considerations for qualitative research on irrational beliefs}

Abstract: Irrational beliefs are a psychological construct proposed by Albert Ellis, which refers to a type of dogmatic and inflexible cognitions that a person maintains about personal values and goals. The main interest in these beliefs is that they generate mental health conditions such as stress, anxiety, and depression, in addition to tending to develop dysfunctional behavior in the person, which makes it difficult to achieve goals. This construct has been studied mostly from a quantitative methodology with results that have allowed to confirm various hypotheses of interest that are properly psychological, specifically for cognitivebehavioral psychology. However, these beliefs have an origin in the dynamism between the individual and society, in addition to presenting some difficulties of a philosophical nature, so studying these beliefs from a qualitative methodology can contribute to sociological studies from a Weberian perspective, and to deepen the fundamental understanding of irrational beliefs, through a fully philosophical reflection, particularly with elements of Stoic philosophy, Husserl's phenomenology, and Kantian imperatives. Theories are approached from different axes regarding psychological, methodological, sociological, and philosophical considerations, for the understanding of the scientific relevance of carrying out research with a qualitative approach and the need to integrate a properly sociological and philosophical reflection in the process.

Keywords: irrational beliefs; qualitative research; sociology; phenomenology.

\section{Introducción}

En el presente ensayo se abordarán las creencias irracionales desde la teoría de Albert Ellis, propuesta a mediados del siglo XX (Ellis, 1962). A la fecha, esta teoría sigue ampliándose y aportando a la psicología cognitivo conductual (Ellis, 2003). El concepto de "creencia irracional" puede entenderse desde otras disciplinas como la lógica, la cual puede aplicar criterios para determinar si una conclusión o creencia es racional o lógicamente válida. Sin embargo, los razonamientos y la conducta de las personas en su cotidianidad no tienden a cumplir las normas racionales; la etiqueta de "irracional" en psicología suele referir a algún tipo de "desviación”, lo cual abarca un amplio "paraguas" de constructos, desde sesgos cognitivos a supersticiones y creencias pseudocientíficas; aunque cabe mencionar que, en tanto psicoterapia, estas creencias se contextualizan especialmente desde la teoría de Ellis (Žeželj y Lazarević, 2019).

El principal interés sobre estas creencias es que generan afecciones a la salud mental, además de ideas y comportamientos disfuncionales que dificultan el logro de metas 
(Ellis et al. 2009). El estudio científico de las creencias irracionales se ha realizado principalmente desde la metodología cuantitativa y con un interés propiamente psicológico, sin embargo, este constructo es de naturaleza cualitativa (Ellis, 2003), dado que la formación y efectos de estas creencias se basan en los presupuestos culturales en la dinámica entre los individuos y la sociedad; es decir, si bien este constructo surge desde la psicología, implica aspectos sociológicos y filosóficos (David, Matu et al. 2019).

El objetivo de este ensayo es presentar la pertinencia de mantener una perspectiva sociológica y filosófica al investigar las creencias irracionales desde una metodología cualitativa. Por esta razón también se busca exponer las teorías sociales y filosóficas que dan sentido al origen y estructura a las creencias, desde un dinamismo social y aspectos filosóficos como la percepción del sujeto, la formulación racional y el carácter imperativo o dogmático de las creencias irracionales. En orden de lograr el objetivo del ensayo, este se estructura en distintos ejes, a saber, el psicológico, metodológico, sociológico y filosófico.

Este ensayo comienza pues, con una descripción de las creencias irracionales y sus aspectos específicamente psicológicos, después, se exponen diferencias entre la metodología cuantitativa y la cualitativa en torno a este constructo, para seguir con el desarrollo de los aspectos sociológicos y filosóficos a propósito de una investigación cualitativa de las creencias irracionales. Al final se busca presentar un entrelazamiento de aspectos que confirme la importancia de mantener consideraciones psicológicas, sociológicas y filosóficas al realizar una investigación de esta naturaleza.

\section{Sobre las creencias irracionales}

Albert Ellis nombró como creencias irracionales a un tipo particular de cogniciones, con base en las verbalizaciones que sus clientes expresaban durante las sesiones de psicoterapia. Estas creencias tienen la característica de ser dogmáticas e inflexibles al enunciarse en forma imperativa como "debo", "estoy obligado" y "tengo que", además de contener consecuencias exageradas y negativas (Ellis, 2003), por ejemplo: "debo conseguir un doctorado o seré un fracasado" o "estoy obligado a ganar la competencia para que mi familia me respete".

Ellis las llamó creencias irracionales desde una perspectiva práctica, ya que estas aparentes obligaciones y consecuencias, no concordaban con la realidad, es decir, no hay evidencia de que la persona tuviera realmente la obligación o necesidad lógica de cumplir esos imperativos, ni de que fueran a tener esas consecuencias negativas (Ellis y Dryden, 1989). 
Estas creencias irracionales tienen un origen social, ya que se derivan de apropiarse dogmática e inflexiblemente de los valores y metas sociales o culturales, y son activadas por un evento o circunstancia que motiva a la persona a formular la creencia (Ellis, 2003). Así, por ejemplo, una meta cultural como puede ser conseguir un grado académico, sumada el evento de leer una nota periodística donde se reporta que los profesionistas con grado académico de licenciatura ganan prácticamente lo mismo que los no graduados, puede generar la creencia irracional: "debo conseguir un doctorado o seré un fracasado, y no tendré un buen ingreso laboral". En este ejemplo, se cuentan con elementos o evidencia para sugerir que conseguir un doctorado pudiera mejorar la posibilidad de tener un buen ingreso laboral, sin embargo, no hay evidencia de que, contar con un doctorado sea la única forma de poder conseguir un buen ingreso laboral, y tampoco muestra la necesidad de ser irremediablemente un fracasado en caso de no contar con ese grado académico.

Ellis identificó la función de las creencias en la filosofía del estoico Epicteto, el cual expresó que, no es lo desafortunado de los eventos desagradables lo que nos perturba, sino nuestras creencias sobre los mismos. De esto último, Ellis planteó el esquema $A B C$, donde "A" es un evento activador, "B" las creencias sobre el evento, y "C" es la consecuencia del evento. Ellis, siguiendo a Epicteto, sugiere que los eventos no generan directamente las consecuencias, sino, las creencias sobre los eventos. Así, por ejemplo, si una persona es despedida (A), y la persona tiene la creencia de que no puede de ninguna forma quedarse sin trabajo, porque sería lo peor que le pudiera ocurrir, además de que no volvería a encontrar un trabajo tan bueno como el que tenía (B), generará como consecuencia perturbaciones como frustración, ira, desesperación, estrés y depresión (C). Estas consecuencias no son generadas directamente por el despido, sino por las creencias irracionales de la persona sobre el despido (B) (Dryden y Still, 1998).

En el ejemplo anterior, las consecuencias generarán en la persona afecciones a su salud mental, pero también afectarán su comportamiento volviéndolo disfuncional (Balkis, 2013; Ellis, 2003). Ellis considera que, por el contrario, se puede tener creencias racionales o funcionales ante los mismos eventos desafortunados, como puede ser, siguiendo el ejemplo anterior, tener la creencia de que: "no quisiera perder mi trabajo, es muy bueno, pero es probable que me despidan". Esta creencia no evitaría que el evento de despido sea desagradable e indeseable, pero prepara a la persona para enfrentar la situación de una forma funcional ante la adversidad (Ellis y Dryden, 1989).

Todas las personas tienden a generar estas creencias irracionales en distintos momentos de su vida, y, como se comentó al principio, generan perturbaciones psicológicas como estrés, ansiedad y depresión, además de originar un comportamiento disfuncional que dificulta el logro de metas (Still y Dryden, 1998). Esto último muestra el evidente interés desde 
la Psicología, sin embargo, para un estudio que comprenda el fenómeno ampliamente, es pertinente, sino que necesario, el aportar una perspectiva social en tanto el origen y resultados de las creencias irracionales, además de un análisis filosófico de la constitución de la propia creencia.

Antes de abordar los aspectos sociológicos y filosóficos, se presentan consideraciones metodológicas a propósito de la diferencia entre un abordaje cuantitativo y cualitativo.

\section{Sobre el estudio cuantitativo y cualitativo de las creencias irracionales}

Ellis, inicialmente se formó como psicoanalista, pero ejerciendo su profesión consideró que el psicoanálisis no resultaba eficiente para ayudar a mejorar la salud mental y comportamiento las personas. Con base en su experiencia clínica fue que desarrolló su teoría cognitivo conductual, la cual incluye el esquema $A B C$ y el constructo de creencias irracionales. Esta teoría, tras algunas modificaciones, terminó por llamarse Terapia racional emotivo conductual (Ellis y Dryden, 1989). Lleva el nombre de "terapia" por tratarse de una teoría enfocada a la psicoterapia, aunque puede comprenderse como una teoría para la investigación científica psicológica con independencia del ejercicio clínico.

Cuando Ellis presentó su teoría en 1958, la psicología era criticada de no cumplir con el rigor científico, el cual, en esa época tenía una imperante forma positivista y cuantitativa. Ellis concordaba con que el método científico era el camino adecuado para el desarrollo del conocimiento, y a la vez, contrario a las críticas, consideraba que la psicología sí constituía una ciencia. Por esta razón realizó y promovió la investigación científica positiva y cuantitativa de sus teorías más allá de la práctica clínica (Ellis y Dryden, 1989). Es importante señalar que, en esa época, la metodología cualitativa no era aceptada por gran parte de la comunidad científica como una metodología rigurosa, y por ende no era válida dentro de criterios positivistas, aunque iba ganando terreno. Esto llevó naturalmente a que los primeros estudios sobre creencias irracionales fueran cuantitativos, aun cuando el constructo es esencialmente cualitativo. Cabe mencionarse para los fines de este ensayo, que la sociología también se emprendió inicialmente desde el positivismo (Weber, 1990), la cual, pretendía construir el conocimiento de lo social con base en un modelo objetivista, inspirado en las ciencias de la naturaleza (Portantiero y de Ipola, 1987).

Los estudios cuantitativos han permitido confirmar teorías que Ellis formuló sobre cómo las creencias irracionales generan perturbaciones psicológicas como ansiedad y depresión; ideas y comportamientos disfuncionales; además de relacionarlas con una disminución del bienestar subjetivo en aspectos como la satisfacción con la vida (Bartucz y 
David, 2019; Chan y Sun, 2020; Stephenson et al. 2018; Turner et al. 2019). Estos estudios han sido exitosos a través de objetivos propiamente cuantitativos como observar la prevalencia de estas creencias en una población, y confirmar las correlaciones o asociaciones con otras variables, por medio de instrumentos validados y el análisis de datos con estadística paramétrica descriptiva e inferencial (Dryden, 2019).

Referente al proceso científico riguroso, cabe mencionarse que la creación o validación de los instrumentos en una población particular, que permiten los estudios cuantitativos de constructos psicológicos, ya supone un trabajo de carácter cualitativo, ya que el instrumento debe tener fiabilidad y validez, lo que implica que se debe ajustar cualitativamente a la población, esto es, a las características culturales complejas como el idioma y conceptos sociales, además de situaciones particulares como el contexto político o hasta condiciones geográficas (Lagunes, 2017).

En el caso de las creencias irracionales, los instrumentos o test para identificar estas creencias en las personas, deben efectivamente discriminar entre una creencia irracional y una que no lo es. Las creencias irracionales suponen contener un imperativo o un sentido inflexible de obligación, pero este no siempre esta expresado textualmente con un "debo". Esto significa que es necesario "buscar el debo", es decir, que se requiere encontrar una forma de expresar en el test una creencia con sentido de obligación (un deber), de manera que, en el momento que la persona la lea o escuche, la identifique como tal, como una obligación y no como una simple preferencia, para entonces, esta persona indique si tiene o no esa creencia (David, DiGiuseppe et al. 2019).

Un ejemplo de lo anterior puede ser la diferencia entre dos formas de expresar una misma creencia con sentido de obligación, pero pudieran entenderse de diferente manera; consideremos una primera creencia: "de ninguna manera puedo perder mi trabajo"; y una segunda: "debo mantener mi trabajo". Aun si en la segunda sí tenemos la palabra "debo", es posible que, en alguna población, la primera frase se interprete como una obligación, mientras que la segunda, que contiene el "debo", se interprete sólo como algo deseable. Por el contrario, en otra población puede que efectivamente, la segunda represente una obligación, mientras que la primera solo una forma de expresar algo deseable.

Confirmar cómo se interpreta el contenido de un test en una población, es parte del proceso de validación, el cual, como se comentó, puede ser entendido como una característica cualitativa de un proceso para la realización de una investigación cuantitativa. Sin embargo, no se pretende decir que la investigación cualitativa de las creencias irracionales sólo tenga utilidad en aportar a investigaciones cuantitativas y específicamente en la creación de instrumentos. Lo que se busca ilustrar con lo anterior, es el interés social y filosófico en el estudio de las creencias irracionales, a saber, el comprender que estas 
creencias tienen los mismos efectos negativos con independencia de la población, pero se expresan de diferente forma y se originan de distintas ideas y circunstancias, dependiendo de las particularidades sociales de la población y la experiencia fenomenológica de los individuos. De hecho, dentro de las investigaciones sobre creencias irracionales, se recomienda para futuras investigaciones el analizar los mecanismos sociales que contribuyen al desarrollo de estas creencias (David, Matu et al. 2019).

\section{Aspecto sociológico en el estudio de las creencias irracionales}

La relación entre las creencias irracionales y la sociedad no se agota en el hecho de que se originan en el individuo por la apropiación dogmática e inflexible de los valores sociales. Tampoco en el otro extremo donde las creencias del individuo son manifestadas o vertidas en la sociedad misma por medio de su comportamiento individual.

De acuerdo con la teoría social de Weber, las acciones de los individuos no son vagos y tenues reflejos particulares o accidentales de la sociedad, ni las acciones del individuo son hechos aislados e intrascendentes, sino todo lo contrario, es el individuo y sus acciones lo que constituye fundamentalmente la sociedad completa. Para Weber, la sociología es entendida como la ciencia de la acción social (Duek, 2009). Con su método individualista, Weber procede a un análisis social desde la acción de uno o varios individuos, explicando cómo el colectivo, o los grandes grupos, pueden reducirse al actuar de las personas particulares, como si estas acciones fueran el átomo de la sociedad (Weber, 1990).

Esta forma comprensiva de ver a la sociedad, implica que la acción social no existe fuera de la subjetividad individual que la constituye. Los conceptos de colectivos como grupos, asociaciones y la sociedad misma, no tienen un sentido especifico, más que el cúmulo de acciones individuales (Duek e India, 2005).

La teoría de Weber es totalmente opuesta a otras teorías clásicas sociales como la de Marx, donde una sociedad es una estructura en la que participan los individuos, pero la suma de estos no la constituye. En la teoría marxista, la acción humana, el comportamiento de las personas, es producto de su inserción en una estructura social y sus prácticas económicas, políticas e ideológicas. Por eso Marx no parte de la persona sino de las relaciones sociales del modo de producción (Marx, 2014). La crítica de Weber a Marx es que no hay ninguna distinción de principio o esencial entre la acción de un individuo y la de muchos individuos (Weber, 1992). Con esta crítica, Weber busca insistir en su teoría donde, en los individuos y sus comportamientos, se puede comprender la sociedad.

Weber advierte que el considerar al conjunto de acciones particulares que constituyen a los grupos que conforman a la sociedad, como entidades independientes y trascendentes 
al individuo, que además lo crean y determinan, es un "falso realismo conceptual" o una "sustancialización" de los conceptos. Esto es atribuirle una esencia ontológica a lo que en realidad es solo una etiqueta o abreviatura para referir a un conjunto de acciones e intereses de individuos (Duek, 2009).

Desde la teoría de Weber, el interés de la sociología va más allá de la ciencia natural ya que trasciende sus leyes que pudieran volver el comportamiento de los individuos en algo predeterminado. Para Weber, la conducta de los individuos, así como los valores, metas y motivaciones, que no están sujetas a las leyes determinantes como en las ciencias naturales, son los protagonistas del estudio sociológico (Duek, 2009).

Las creencias irracionales en una población en particular, representan solo una parte de la idiosincrasia de la sociedad a la que pertenecen, pero una de suma relevancia, ya que esta parte se constituye por los valores y metas ideales de sus miembros, es decir, forman la base y motivaciones del mantenimiento y desarrollo de esa sociedad, y, siguiendo a Weber, esto genera una evolución o dialéctica entre el individuo y la sociedad, es decir, entre la formación y comportamiento de cada persona y la acción social. La sociedad influye en las creencias de los individuos, los cuales, al asimilarlas con las características indeterminables de las circunstancias de cada uno, estos terminan por estructurar nuevas versiones de la sociedad, la cual a su vez influirá en la formación de nuevos individuos, en un ciclo evolutivo constante. Estudiar pues las creencias irracionales de los individuos con un enfoque social cualitativo, permite aprovechar la investigación para la comprensión de una parte del origen, estado actual y desarrollo de una sociedad.

\section{Aspecto filosófico}

La filosofía es una disciplina que tiene como objetivo general comprender la realidad y lo hace por medio de la razón. Por esto es natural que el avance científico, basado en la observación y experimentación, haya atendido y explicado problemas que antes eran de carácter filosófico, por ejemplo, conocer los elementos que componen a los seres vivos; y cuál es la forma y movimiento de los astros. Sin embargo, prevalecen cuestiones que, por el momento, siguen precisando de una perspectiva filosófica, por ejemplo, las deliberaciones éticas y, propiamente sobre el tema de este ensayo, la formación de las creencias irracionales y la influencia que tienen en el comportamiento. Por medio de la metodología cuantitativa se pueden medir y relacionar con sus efectos, pero no explica cómo es que dos personas con perfiles y contextos semejantes pueden desarrollar creencias diferentes sobre las mismas cosas. No es extraño pues, que Ellis, un académico contemporáneo occidental, haya utilizado una reflexión estoica para su esquema $\mathrm{ABC}$, como se describió al principio de este ensayo. 
En realidad, el ejercicio de la filosofía no se contrapone con la ciencia de ninguna forma. La ciencia se puede usar para resolver dudas que emergen de la filosofía, a la vez que la filosofía se ejercita en el método científico, por ejemplo, al desarrollar una hipótesis de investigación, ya que implica el relacionar conceptos por medio de una argumentación razonable que permite sugerir un posible efecto en la realidad. Otro ejemplo que puede identificarse como un ejercicio filosófico es el momento de discutir los resultados obtenidos, puesto que implica una interpretación, es decir, agregar información que no está necesariamente contenida en los datos empíricos, sino, en una comprensión por parte del investigador que tiene una visión holística y vivencial de los fenómenos, que le permiten interpretar coherentemente los datos con la realidad.

Esto significa que la investigación cuantitativa de las creencias irracionales puede alcanzar sus objetivos, por ejemplo, medir la prevalencia de creencias irracionales en una población. Sin embargo, la investigación cualitativa que integre un enfoque filosófico, vuelve asequible una comprensión del fenómeno más amplia. Elementos filosóficos relacionados a las creencias irracionales son la filosofía estoica aplicada en el esquema ABC (Dryden, 2019), y, además, se puede identificar el proceso fenomenológico descrito por Husserl, el cual parece constituir las creencias en los individuos; y también las características de los imperativo que aparecen en la filosofía de Kant.

\subsection{Influencia estoica}

Como se comentó en la primear parte de este ensayo, Ellis consideró lo dicho por el estoico Epicteto para formalizar su esquema $A B C$, a saber, que no es lo desafortunado de los eventos desagradables lo que nos perturba, sino nuestras creencias sobre los mismos. Es decir, son las creencias sobre lo que nos ocurre, lo que influye en cómo nos sentimos al respecto, y no lo que ocurrió en sí. Pero cabe aclarar que tanto Epicteto como Ellis no sugieren que lo adecuado sea aspirar a desarrollar creencias que solo generen emociones 0 sentimiento agradables y positivos. Por el contrario, es adecuado sentirse mal ante eventos desagradables.

Específicamente en la teoría de Ellis, se explica que es saludable experimentar consecuencias negativas como rechazo, incomodidad y tristeza, ya que nos permiten identificar lo que no es agradable para nosotros y promueve el desarrollo de un comportamiento funcional que favorece el logro de metas. Lo que se busca evitar es generar perturbaciones psicológicas como la ira, estrés y depresión, las cuales generan dificultades a la persona para identificar claramente el problema y promueve un comportamiento disfuncional (Ellis y DiGiuseppe, 1993).

Desde la filosofía estoica se acepta que los seres humanos estamos naturalmente inclinados a ciertas cuestiones convencionales como la salud o la riqueza y tratamos de evitar 
otras como el ser odiados o la muerte de nuestros allegados. No obstante, incluso la muerte de un ser querido, es un evento que, dependiendo la creencia, puede generar un comportamiento disfuncional o funcional; estos dos comportamientos se denominan en la filosofía estoica como "pasiones" o "buenas pasiones" respectivamente. Para ilustrar lo anterior, podemos ejemplificar con un padre que se entera que su hijo tiene cáncer, su natural rechazo a la muerte le llevará a reaccionar, pero sus creencias pueden llevarlo a generar pasiones de miedo y pánico, volviéndolo ineficiente para atender a su hijo de la mejor forma; en cambio su creencia pudiera llevarlo a generar buenas pasiones de voluntad, permitiéndole tener "cabeza fría" y actuar de mejor forma para el bienestar de su hijo (Ortiz Delgado, 2018).

Lo anterior significa que no se niega lo indeseable de algunos eventos o lo desagradable de algunas circunstancias. Lo que se aspira es al desarrollo de una perspectiva de la realidad (creencias) que favorezca al bienestar de la persona. Desde la filosofía estoica se expresa en forma de alcanzar la virtud de la felicidad, lo que incluye aceptar las cosas que son naturales o no dependen del sujeto; y desde la psicología de Ellis, a mantener la salud mental evitando creencias que desarrollen perturbaciones psicológicas.

En una investigación cualitativa sobre creencias irracionales, reflexionar a partir de la filosofía estoica permite advertir la importancia de distinguir en el discurso de un participante, las cosas que rechaza de forma natural, mas no desde una postura irracional, por ejemplo, las expresiones de tristeza que bien pueden ser funcionales y no deberían confundirse con sintomatología depresiva; además de las actitudes que pudieran parecer positivas u optimistas pero que implican una negación de lo natural y pudieran constituir una creencia irracional.

\subsection{El proceso fenomenológico en la formación de creencias}

La filosofía estoica explica que las creencias sobre un evento son las generadoras de las emociones y no los eventos en sí. Pero no explica por qué se pueden tener distintas creencias sobre un mismo evento, en especial cuando el evento es claro y distinto, es decir, que hay una plena comprensión del evento y lo que implica.

Parece tener sentido que dos personas tengan creencias distintas sobre eventos distintos. Por ejemplo, consideremos el posible escenario de dos personas que fueron despedidas de sus trabajos. La primera tiene ofertas de trabajo que no había considerado antes porque ya tenía un trabajo, mientras que la segunda persona sufrió muchas dificultades para conseguir el trabajo que tenía y nunca le ofrecieron otras oportunidades. En este caso, parece intuitivo considerar que la segunda persona, si la despiden, activará creencias negativas sobre el despido, llevándola a desarrollar estrés y angustia, además de un comportamiento conflictivo o disfuncional; mientras que la primera persona tendría creencias y actitudes optimistas y funcionales. Sin embargo, también es posible que, aun en el 
escenario planteado, sea la primera persona la que desarrolle creencias irracionales y no la segunda. Si se diera esto último, se pudiera sospechar que tal vez hay algo más de fondo que explica esto, como pudiera ser que la primera persona tiene muchas deudas económicas mientras que la segunda no, y de ahí que la segunda, pese a sus dificultades en conseguir un empleo, no tenga elementos que la presionen a desarrollar creencias irracionales, mientras que la primera persona, pese a tener un escenario más favorable, sí está presionada u obligada a tener un trabajo. Sin embargo, también es posible que la primera persona no tenga deudas y esté en una circunstancia favorable en todo sentido, y, aun así, desarrollar creencias irracionales. Sea cual sea el caso, lo que se busca exponer con el ejemplo anterior, es que las creencias pueden estar influenciadas por detalles que no son evidentes.

En la teoría de Ellis sobre las creencias irracionales, se ha detectado que estas se originan con relación a los valores y metas culturales, pero no explica por qué algunos las generan y otros no. En el proceso psicoterapéutico se puede indagar sobre cuál es el origen de las creencias en una persona en particular, sin embargo, aún si encontraran las circunstancias de donde emerge una creencia de un cliente, eso constituirá un caso particular en una persona, que no representa una norma con la cual se pueda predecir que otra persona, con esas circunstancias, también vaya a generar esa creencia.

Parece que, en realidad, la formación de creencias irracionales no obedece a un proceso material o social determinado, sino, a un proceso fenomenológico que consta de la experiencia compleja de un sujeto ante la realidad. Esta experiencia no se deduce lógicamente de las características materiales o contextuales, sino de una interpretación del sujeto (Martínez Guerrero, 2004).

La fenomenología fue desarrollada por Edmund Husserl, y en la reflexión desde esta perspectiva filosófica se aplica un proceso llamado reducción fenomenológica, el cual consiste, en tratar de poner un evento de la realidad "entre paréntesis", es decir, sacarlo un momento de todo lo que lo rodea y tratar de contemplar solo ese evento o cosa, con el fin de intentar comprender sólo ese fenómeno particular (Martínez Guerrero, 2004).

Este tipo de análisis trata de pensar un objeto o evento de forma aislada. Por ejemplo, al hacer una reducción fenomenológica de la experiencia de contemplar un árbol, se intenta "poner entre paréntesis" al árbol y dejar fuera todo lo demás, como sus raíces bajo la tierra y el sol que necesita para crecer; no porque se niegue la existencia o relación de las raíces y el sol con el árbol, o se dude de estos, sino, porque al practicar esta reducción se busca cerrar el objeto o evento de todo juicio o relaciones con otras cosas (Martínez Guerrero, 2004), para dejar de forma aislada el objeto de interés, que en este caso es el árbol. Sin embargo, sí puede incluir en cierta forma al sol u otros elementos, ya que ese fenómeno particular de contemplar un árbol en específico y que fue puesto entre paréntesis, puede incluir la noción 
de luminosidad y tibiez, aunque en otros casos de contemplación de un árbol, es decir, otros fenómenos, puede no tener estos elementos y, en cambio, contener los de oscuridad y frialdad; todo dependerá del fenómeno en particular. También es posible que el fenómeno que se busque reducir poniéndolo entre paréntesis no sea el árbol, sino un paisaje completo. Lo que es importante en la reducción es, en el caso del árbol, no brincar al paisaje completo, o, en el caso de paisaje, no omitir los demás elementos y considerar solo un árbol.

La reducción fenomenológica es pues, el método radical por el cual el sujeto se capta a sí mismo en forma pura, con la vida de conciencia pura que le es propia, vida en y para la cual la totalidad del mundo objetivo existe para este (Carlson, 2014). Generalmente concordamos entre sujetos en cómo está constituida la realidad ya que todos los humanos percibimos prácticamente de la misma forma, con los mismos sentidos. Sin embargo, hay variaciones que derivan de las particularidades únicas de cada persona y sus circunstancias, y estas variaciones en cómo se experimentan los fenómenos, son variaciones en la realidad que sólo tienen pleno sentido en el sujeto que lo experimenta y pierde sentido cuando se intenta que la experiencia o realidad de un sujeto sea la misma que en otro.

Cabe advertir que la reducción fenomenológica implica una desconexión de la percepción del objeto de interés con el resto del mundo e ideas; y la suspensión de juicios, dejando la vivencia pura de la experiencia del fenómeno que se contempla (Martínez Guerrero, 2004). De esto último, resulta que no es viable identificar una creencia irracional durante la reducción fenomenológica, dado que una creencia expresa la relación de un evento con sus circunstancias. Pero el proceso de reducción permite al sujeto describir y comprender lo que el evento realmente le representa, con lo cual, posteriormente podrá relacionarlo con sus creencias.

\subsection{Forma racional de las creencias}

Si bien con la consideración estoica sobre las creencias y la reducción fenomenológica se puede investigar sobre el origen y efecto de las creencias, no queda claro por qué una persona mantendría una creencia que aparenta ser claramente irracional, y, además, por qué le otorgaría un carácter imperativo en forma de obligaciones como "debo" y "tengo que". Por ejemplo, una persona puede creer que: "quisiera graduarme este año, porque si no demostraré que no sirvo para nada", sin creer de forma imperativa que: "debo graduarme este año, porque si no demostraré que no sirvo para nada"; la diferencia entre estas dos versiones del ejemplo es que en la primera se expresa como preferencia utilizando "quisiera" y la segunda como imperativo usando "debo". Cabe señalar que, de acuerdo a la teoría de Ellis, en la primera versión de este ejemplo de creencia, al decir "quisiera" la vuelve una creencia racional, pero solo la primera parte, ya que después se junta con una creencia irracional catastrófica al creer que, de no lograr su meta, demostrará que no sirve para nada; 
mientras que, en la segunda versión, al usar "debo" desde el principio, se trata de dos creencias irracionales juntas (DiGiuseppe et al. 2017).

Lo anterior significa que es razonable que una persona, al no tener evidencias o seguridad sobre algo, genere creencias en forma de preferencias, sugerencias o probabilidades. Pero darle forma imperativa a algo sin evidencia de que debe ser así, denota el carácter irracional. Sin embargo, es importante advertir aquí que lo racional no es necesariamente basarse solo en evidencia científica, ya que, para empezar, no es posible conocer todo sobre nuestro actuar cotidiano, por ejemplo, el conocimiento nutricional de nuestros alimentos, la mecánica y tecnología de todos los aparatos o máquinas que utilizamos, las teorías sociales y pedagógicas al momento de convivir con personas e ir a la escuela, etc. Al final las personas actúan de acuerdo a lo que están convencidas que funciona, por ejemplo, hay personas que no comen huevo por la creencia popular de que incrementa los niveles de colesterol, aun cuando en diferentes estudios se informa que el consumo de huevo no está relacionado con un mayor nivel de colesterol, ni con la aparición de enfermedades cardiovasculares, las cuales se relacionan con otros factores como la actividad física (Oriondo Gates et al. 2013). Una dificultad agregada, es que aun cuando se buscan disipar dudas acudiendo con especialistas que se basan en el conocimiento científico, estos pueden tener posturas contradictorias.

Actuar o mantener una creencia racional implica la dificultad de creer que se tiene conocimiento fiable con el cual se basa el comportamiento. Cuando una creencia se basa en el discurso de una disciplina como el conocimiento científico se le puede llamar una "racionalidad discursiva"; pero a veces el actuar se basa en simplemente la creencia de que algo funciona, por tanto, se puede considerar que es razonable hacer lo que se cree que funciona, a esto se le conoce como "minirat" por ser la racionalidad mínima por la cual se procede a un actuar (Still, 2009). La dificultad radica en que, actuar con base en un conocimiento científico puede parecer más racional que actuar con base en una mera creencia, pero las personas rara vez son el grupo de científicos que han realizado la investigación científica y comprobado el mencionado conocimiento científico, por lo que al final las personas confían en la revista, nota periodística o dato compartido por una amistad, donde afirman que cierta información está comprobada científicamente. Esto no es negar la efectividad del método científico identificándolo con una mera creencia o suposición, sino, que la persona en concreto no puede tener la certeza absoluta de que la información compartida presuntamente generada por un proceso científico, efectivamente lo es, y, por tanto, termina por confiar y actuar con base en lo que considera es verdad. Lo anterior busca ilustrar la dificultad de aspirar a actuar racionalmente solo con bases científicas, puesto que parece difícil o imposible salir por completo del actuar con base en creencias. 


\subsection{Carácter imperativo de las creencias}

Dado que la racionalidad de una creencia puede venir del discurso de una disciplina o simplemente de lo que asumimos como verdad porque tal vez nos parece evidente, parece claro que el carácter imperativo puede devenir de esta misma razón. Ellis sostiene que las creencias irracionales se originan de la apropiación dogmática de los valores culturales. Sin embargo, aun en las creencias o posturas dogmáticas se puede exigir coherencia o lógica, por ejemplo, una persona puede creer dogmáticamente que el capitalismo es el mejor sistema económico posible, y, si bien es dogmático en esto, puede igualmente contar con una serie de proposiciones con la que fundamente su creencia, aún si estas proposiciones son falsas, falaces o incorrectas. Este ejemplo de creencia dogmática que busca ser fundamentada, se distingue de una postura propiamente lógica, en que, si a la persona dogmática se le presenta evidencia de que sus proposiciones son incorrectas, por su dogmatismo, podría aferrarse a sus proposiciones que dan sentido a su creencia y considerará como falsas a la evidencia presentada.

De lo anterior se presenta un conflicto entre las evidencias en la realidad y lo racional. Es decir, las creencias denominadas como irracionales según la teoría de Ellis, pudieran ser argumentos con una estructura lógicamente válida, por ejemplo, "los inútiles no deben intentar sobresalir porque empeoran las cosas; por tanto, yo, que soy un inútil y no quiero empeorar las cosas, no debo intentar sobresalir". Esta evaluación global y negativa de uno mismo es un tipo de creencia irracional de acuerdo a la teoría de Ellis (Dryden, 2019), pero no parece ser irracional en realidad, de hecho, es un razonamiento válido que se sigue lógicamente de las proposiciones. Sin embargo, difícilmente se tendrá evidencia en la realidad que fundamente la generalización negativa de "yo soy un inútil".

Para resolver la posibilidad de que una creencia imperativa, que de acuerdo con Ellis sería irracional, pueda ser a la vez una creencia lógicamente válida y por tanto no sería irracional, conviene considerar dos formas imperativas descritas por Immanuel Kant. Este autor describe el concepto de imperativo para atender el problema de la ética, es decir, el cómo debería de actuar una persona.

Kant parte de considerar que el humano es un ser motivado por la razón, pero a la vez es un ser finito, siendo esta finitud una motivación diferente a la razón. La existencia de estas dos fuentes motivacionales tiene como resultado, el que la irracionalidad sea siempre una posibilidad abierta al obrar humano. Por este motivo, una acción puede fundamentarse en principios que sean racionales, aunque esta la posibilidad de que estos principios sean requerimientos sugeridos libremente por el propio sujeto; en cualquier caso, estos principios o requisitos expresan un "tener que", y es este "tener que" lo que constituye un imperativo (Kant, 1921; Placencia, 2016). 
Una vez definido lo que es un imperativo, Kant introduce dos formas de este, a saber, hipotéticos y categóricos. Los imperativos categóricos mandan de modo incondicionado, es decir, sin una condición particular, debiendo ser cumplidos sin importar la circunstancia, por ejemplo, no mentir. Kant argumenta que no mentir es un imperativo que debe cumplirse sin importar las condiciones de la situación, por tanto, es categórico, es decir, incondicionado. Mientras que los imperativos hipotéticos son condicionados, formulan un deber requerido en caso de presentarse cierta condición, por ejemplo, "estudia para el examen", en este caso, este imperativo no aplica para cualquier momento o situación, ya que sólo tiene sentido en caso de que se vaya a presentar un examen, por tanto, es hipotético o condicionado. La diferencia entre imperativos categóricos e hipotéticos no es su forma lingüística, sino, el que estén condicionados o no a una circunstancia (Placencia, 2016).

Cabe mencionar que, además de la característica de incondicionado, en tanto contenido, los imperativos categóricos representan una carga moral, es decir, la razón por la que son incondicionados es que obedecen a un "tener que" o "deber" que la persona considera debería de ser realizado con independencia de los detalles particulares, en orden de obrar éticamente (Cabrera, 2017; Kant, 1921). Por ejemplo, entregar una cartera extraviada, pudiera ser un imperativo categórico, si se considera como un "deber" sin importar las condiciones que pudieran darse, por ejemplo, si dentro de la cartera hay poco o mucho dinero; o si la persona que la encontró pudiera tomar el contenido o no. Por otra parte, un ejemplo de imperativo hipotético puede ser "si quiero mostrarme como una persona honesta ante los demás que notaron que me encontré una cartera, debo intentar devolverla". Aquí se observa que hay condiciones particulares que fundamentan el "deber" distintas a la ética o moral, en este caso, que hubo personas que notaron el hallazgo de la cartera, y el objetivo de aparentar honestidad. Estos imperativos hipotéticos no tienen esta carga moral, en el ejemplo recién dado de la cartera, el imperativo hipotético se muestra con un fin deshonesto o hipócrita, pero bien puede ser ajeno a juicios morales y ser sólo técnicos, por ejemplo, "debo mandar reforzar mi mochila para que no se siga desgarrando".

La formulación de imperativos de acuerdo con Kant, identifican que el origen del "deber" o imperativo, deviene de una carga moral o de una serie de condiciones que deben cumplirse en orden de lograr algún fin particular. Estos pueden estructurarse desde un ejercicio lógico y de rigor ante evidencia empírica, o bien, desde una argumentación con proposiciones subjetivas. De esta forma se puede mantener un seguimiento lógico de ideas, sin necesidad de basarse en la realidad, lo cual puede ser identificado como irracional, pero no por un error lógico, como se comentó anteriormente en la descripción de las creencias irracionales según Ellis, sino, desde una perspectiva práctica, justamente al no fundamentarse en la evidencia empírica. 
De esta forma, las creencias que parten de la experiencia fenomenológica adquieren su carácter lógico e imperativo, aún si pueden ser denominadas como irracionales en la teoría de Ellis. Esto es, que la apropiación dogmática de un valor, constituye la formulación de imperativos categóricos e hipotéticos, es decir, la persona formula "deberes" con el fin de lograr una meta, basado en proposiciones de orden moral o técnicas, sin importar si estas vienen con algunos elementos de evidencia empírica o de otras creencias y conceptos culturales. De esta forma, la persona que sostiene estas creencias dogmáticas, no necesariamente incurre en errores estrictamente lógicos, por el contrario, puede formular razonamientos válidos que, al ser lógicos, teóricamente pueden constituir la única forma lógica de lograr cierto objetivo, pasando así a volverse imperativos. Esto no contradice la teoría de Ellis, pero permite advertir que la categoría de irracional parte de un sentido práctico sobre la vivencia cotidiana, y no de un análisis lógico del argumento que puede constituir una creencia.

\section{Conclusiones}

La investigación científica con el objetivo de conocer, comprender y predecir la realidad, requiere una estructura compleja de conocimientos previos que posibilitan la formulación de hipótesis nuevas y estudios para comprobarlas. Por otra parte, tal vez la virtud más grande de la ciencia, sea que está permanentemente abierta a la corrección o ajuste de conocimientos, aun de los que se consideraban ya probados o esclarecidos.

En ese sentido, considero que la investigación de las creencias irracionales desde una metodología cualitativa incluyendo una perspectiva social y filosófica, constituye una oportunidad de revisar el sentido y significado que tiene ese tipo de creencias tanto para el individuo como en la sociedad, ampliando así la discusión sobre los resultados cuantitativos y las consideraciones teóricas para nuevos problemas de investigación.

Es conveniente notar la relevancia de aplicar efectivamente un tratamiento o análisis sociológico y filosófico al momento de estudiar cualitativamente las creencias irracionales. Es decir, trascender una posible lista de pasos definidos en el procedimiento de realizar una entrevista semiestructurada, y aplicar un proceso de reflexión particular a cada caso, orientado por las teorías sociales y filosóficas que pueden ayudar a clarificar los fenómenos complejos de la realidad, como suelen ser los relacionados con los seres humanos.

Las creencias irracionales constituyen pues, un fenómeno complejo donde se intersecan aspectos psicológicos al influir en el comportamiento y salud mental; teorías sociológicas que buscan explicar la relación entre el individuo y la sociedad; además de perspectivas filosóficas que permiten sugerir el origen y estructura de las creencias. 
En el presente ensayo se aspiró a presentar la pertinencia de mantener una perspectiva sociológica y filosófica al investigar las creencias irracionales desde una metodología cualitativa, además de presentar la teoría sociológica de Weber y los elementos filosóficos sobre el efecto de las creencias en las emociones, desde el estoicismo, la fenomenología de Husserl y los imperativos Kantianos. Todo lo anterior con la intención de orientar las posibles investigaciones y aportar a la discusión de los datos obtenidos.

El autor de este trabajo, siendo estudiante en la Maestría en Psicología con orientación en calidad de vida y salud, de la Universidad de Guadalajara, agradece al Consejo Nacional de Ciencia y Tecnología (CONACYT) por la beca percibida durante el periodo en que realizó el presente ensayo; también, particularmente agradece a su tutor, el doctor Felipe Santoyo Telles, por su apoyo y orientación.

\section{Referencias bibliográficas}

Balkis, M. (2013). Academic procrastination, academic life satisfaction and academic achievement: The mediation role of rational beliefs about studying. Journal of Cognitive and Behavioral Psychotherapies, 13(1), 57-74.

Bartucz, M. B., y David, D. O. (2019). Irrational beliefs at country level functioning: A crosscultural extension of the cognitive-behavioral model. Journal of Evidence-Based Psychotherapies, 19(1), 1-26. https://doi.org/10.24193/jebp.2019.1.1

Cabrera, C. (2017). La apropiación husserliana del Imperativo Categórico. Arete, 29(1), 2958. https://doi.org/10.18800/arete.201701.002

Carlson, S. (2014). Reducción monadológica y reducción fenomenológica: el problema de la reducción en Husserl y en Richir. Eikasia: revista de filosofía, 57, 239-256.

Chan, H. W. Q. y Sun, C. F. R. (2020). Irrational beliefs, depression, anxiety, and stress among university students in Hong Kong. Journal of American College Health. https://doi.org/10.1080/07448481.2019.1710516

David, D., DiGiuseppe, R., Dobrean, A., Păsărelu, C. R. y Balazsi, R. (2019). The Measurement of Irrationality and Rationality. En M. Bernard y W. Dryden (Eds.), Advances in REBT (pp. 79-100). Springer International Publishing. https://doi.org/10.1007/978-3-319-93118-0_4

David, D. O., Matu, S. A., Podina, I. R. y Predatu, R. M. (2019). Future Research Directions for REBT. En M- Bernard y W. Dryden (Eds.), Advances in REBT (pp. 121-146). 
Springer International Publishing. https://doi.org/10.1007/978-3-319-93118-0_6

DiGiuseppe, R., Leaf, R., Gorman, B. y Robin, M. W. (2017). The Development of a Measure of Irrational/Rational Beliefs. Journal of Rational-Emotive \& Cognitive-Behavior Therapy, 36(1), 47-79. https://doi.org/10.1007/s10942-017-0273-3

Dryden, W. (2019). The Distinctive Features of Rational Emotive Behavior Therapy. En M. E. Bernard \& W. Dryden (Eds.), Advances in REBT. Springer International Publishing. https://doi.org/10.1007/978-3-319-93118-0

Dryden, W. y Still, A. (1998). REBT and rationality: Philosophical approaches. Journal of Rational - Emotive and Cognitive - Behavior Therapy, 16(2), 77-99. https://doi.org/10.1023/A:1024920326970

Duek, M. (2009). Individuo y soociedad: perspectivas teórico-metodológicas en la Sociología Clásica. Argumentos: Estudios Críticos de la Sociedad, 22(60), 9-24.

Duek, C. e India, G. (2005). Individualismo metodológico y concepción del Estado en Max Weber. La acción individual como productora del orden político. Universum. Revista de humanidades y ciencias sociales, 27.

Ellis, A. (1962). Reason and emotion in psychotherapy. Lyle Stuart, Inc.

Ellis, A. (2003). Early Theories and Practices of Rational Emotive Behavior Therapy and How They Have Been Augmented and Revised During the Last Three Decades. Journal of Rational-Emotive and Cognitive-Behavior Therapy, 21(3-4), 219-243. https://doi.org/10.1023/A:1025890112319

Ellis, A., David, D. y Lynn, S. J. (2009). Rational and Irrational Beliefs: A Historical and Conceptual Perspective. En D. David, S. Lynn y A. Ellis (Eds.), Rational and Irrational Beliefs: Research, Theory, and Clinical Practice (pp. 3-22). Oxford University Press. https://doi.org/10.1093/acprof:oso/9780195182231.003.0001

Ellis, A. y DiGiuseppe, R. (1993). Are inappropriate or dysfunctional feelings in rationalemotive therapy qualitative or quantitative? Cognitive Therapy and Research, 17(5), 471-477. https://doi.org/10.1007/BF01173058

Ellis, A. y Dryden, W. (1989). Práctica de la Terapia Racional Emotiva. Springer Publishing Company.

Kant, E. (1921). Fundamentación de la Metafísica de las Costumbres. [P. M. Rosario Barbosa (ed.)].

Lagunes, R. (2017). Recomendaciones sobre los procedimientos de construcción y validación de instrumentos y escalas de medición en la psicología de la salud. Psicología y Salud, 27(1), 5-18. 
Martínez Guerrero, J. M. (2004). En torno a la "Reducción” en Husserl. Isla de Arriarán, 23, 381-398.

Marx, C. (2014). El capital: Crítica de la economía política (4a ed.). Fondo de Cultura Económica.

Oriondo Gates, R. L., Bernui Leo, I., Valdivieso Izquierdo, L. R. y Estrada Menacho, E. (2013). Relación entre colesterol dietario, consumo de huevo y perfil lipídico en adultos aparentemente sanos, según grupos de edad. Anales de la Facultad de Medicina, 74(1), 27. https://doi.org/10.15381/anales.v74i1.2036

Ortiz Delgado, F. M. (2018). Los Sentimientos Ante La (Nada Terrible) Muerte En La Filosofía Estoica. Stoa, 9(17), 7-25. https://doi.org/10.25009/s.2018.17.2532

Placencia, L. (2016). Kant y el problema de la Razón Instrumental. Revista de Filosofia: Aurora, 28(44), 409-431. https://doi.org/10.7213/aurora.28.044.DS02

Portantiero, J. C. y de Ipola, E. (1987). Estado y sociedad en el pensamiento clásico. Cántaro.

Stephenson, E., Watson, P. J., Chen, Z. J. y Morris, R. J. (2018). Self-Compassion, SelfEsteem, and Irrational Beliefs. Current Psychology, 37(4), 809-815. https://doi.org/10.1007/s12144-017-9563-2

Still, A. (2009). Rationality and Rational Psychotherapy: The Heart of REBT. D. David, S. Lynn y A. Ellis (Eds.), Rational and Irrational Beliefs: Research, Theory, and Clinical Practice (pp. 23-48). Oxford University Press. https://doi.org/10.1093/acprof:oso/9780195182231.003.0002

Still, A. y Dryden, W. (1998). The intellectual origins of rational psychotherapy. History of the Human Sciences, 11(3), 63-86. https://doi.org/10.1177/095269519801100304

Turner, M. J., Carrington, S. y Miller, A. (2019). Psychological distress across sport participation groups: The mediating effects of secondary irrational beliefs on the relationship between primary irrational beliefs and symptoms of anxiety, anger, and depression. Journal of Clinical Sport Psychology, 13(1), 17-40. https://doi.org/10.1123/jcsp.2017-0014

Weber, M. (1990). Ensayos sobre metodología sociológica (2a ed.). Amorrortu.

Weber, M. (1992). El problema de la irracionalidad en las ciencias sociales (2a ed.). Tecnos. Žeželj, I. y Lazarević, L. B. (2019). Irrational beliefs. Europe's Journal of Psychology, 15(1), 1-7. https://doi.org/10.5964/ejop.v15i1.1903 